\title{
Modelagem de Engenharia da Cognição: Levantando o Cognoma Humano
}

\author{
Carla Verônica M. Marques ${ }^{1}$, Carlo E.T. Oliveira ${ }^{1}$, Claudia L.R. Motta ${ }^{1,2}$ \\ 1Instituto Tércio Pacitti de Aplicações e Pesquisas Computacionais \\ Universidade Federal do Rio de Janeiro (UFRJ) \\ CEP 21941-916 - Rio de Janeiro - RJ - Brasil \\ ${ }^{2}$ Programa de Pós-Graduação em Informática- PPGI/UFRJ \\ Universidade Federal do Rio de Janeiro (UFRJ) \\ Rio de Janeiro, RJ - Brasil. \\ \{carla.veronica, carlo, claudiam\}@nce.ufrj.br
}

\begin{abstract}
To study human cognition is to increase knowledge about ourselves and open new possibilities for human improvement. Cognition is the mechanism by which all learning, reasoning and reflection on any knowledge is realized. Understanding cognition is a crucial path for fair and equitable access to knowledge and understanding of the world around us. The creation of cognitive models is a complex challenge that involves an interdisciplinary effort, constituting a vast field of research to be explored. The development of solid cognitive models can enable a whole new aspect of the human-machine interface. The computer will go from a luxury accessory to an effective and powerful instrument in the hands of teachers, making your work more productive and less frustrating.
\end{abstract}

Resumo. Estudar a cognição humana é aumentar o conhecimento sobre nós mesmos e abrir novas possibilidades para o aperfeiçoamento humano. A cognição é o mecanismo pelo qual se realiza todo o aprendizado, raciocínio e reflexão sobre qualquer conhecimento. $O$ entendimento da cognição é um caminho crucial para haver um acesso justo e igualitário ao conhecimento e à compreensão do mundo que nos cerca. A criação de modelos cognitivos é um desafio complexo que envolve um esforço interdisciplinar, constituindo um vasto campo de pesquisa a ser explorado. O desenvolvimento de modelos cognitivos sólidos pode viabilizar todo um novo aspecto da interface homemmáquina. O computador passará de um acessório de luxo para um instrumento eficaz e poderoso nas mãos dos professores, tornando o seu trabalho mais produtivo e menos frustrante.

\section{Importância e benefícios de modelos cognitivos}

Estudar a cognição humana é aumentar o conhecimento sobre nós mesmos e abrir novas possibilidades para o aperfeiçoamento humano. A profundidade da mente humana é ainda uma fronteira pouco explorada, mas de grande significado para o avanço individual e da sociedade. Sendo um assunto de grande complexidade, é essencial que 
se produzam simplificações que permitam que se lide de maneira sucinta e racional com o processo cognitivo.

Um modelo de engenharia tem por premissa que se entenda o funcionamento do objeto estudado como uma máquina. O modelo de engenharia da cognição permitirá que se entenda melhor a cognição através de um sistema que apresenta um desenvolvimento semelhante ao processamento humano. A modelagem de engenharia resulta em sistemas que podem ser facilmente implementados em uma forma computacional.

Um modelo cognitivo pode acompanhar o desenvolvimento do entendimento humano e permitir localizar os pontos onde o processo de aprendizagem resultou em sucesso ou fracasso. A implementação deste modelo em forma computacional pode aprender e adquirir em grande profundidade o conhecimento do sistema cognitivo humano, interpretando indícios obtidos da interação homem-máquina.

O desenvolvimento de modelos cognitivos sólidos pode viabilizar todo um novo aspecto da interface homem-máquina. As interfaces correntes ainda encaram o usuário como um estranho. $\mathrm{Na}$ medida que a máquina consegue sincronizar com o processo cognitivo, o computador se torna uma nova prótese. A cognição então passa a informar o computador das oportunidades que se apresentam para que este apresente uma intervenção coerente e tempestiva.

\section{Justificativa e contribuição para a temática}

A cognição é o mecanismo pelo qual se realiza todo o aprendizado, raciocínio e reflexão sobre qualquer conhecimento. Também nela se encontra todo obstáculo ao entendimento quando algum mecanismo ou procedimento cognitivo não está perfeitamente funcional. $\mathrm{O}$ entendimento da cognição é um caminho crucial para haver um acesso justo e igualitário ao conhecimento e à compreensão do mundo que nos cerca.

A criação de modelos cognitivos é um desafio complexo que envolve um esforço interdisciplinar, constituindo um vasto campo de pesquisa a ser explorado. O estudo da cognição envolve diversas áreas como a psicologia, a fonoaudiologia, a psicopedagogia, a linguística, a lógica/matemática entre outras. Este conjunto de campos, nem todos sendo ciências exatas, requer um esforço criativo no engendramento de soluções e heurísticas que permitam mapear assertivas filosóficas em soluções computáveis.

Um modelo cognitivo computacional é um instrumento valioso no processo educacional, capaz de nortear estratégias de ensino e auxiliando na avaliação das competências auferidas no processo. Modelar a cognição significa ir além do típico paradigma estímulo-resposta para se avançar na introspecção dos mecanismos internos da aprendizagem. A máquina cerebral oferece paradigmas ainda inexplorados que irão aumentar o arsenal de esquemas disponíveis para solução de problemas computacionais. Em contrapartida, o modelo cognitivo computacional se tornará uma ferramenta importante na análise e entendimento dos problemas de aprendizagem. Consequentemente, toda esta informação disponível, trará à tona as reais falhas do processo de ensino e a oportunidade de se intervir com conhecimento de causa. 


\section{O propósito do desafio de modelagem cognitiva}

O desafio de modelagem da cognição propõe a construção de sistemas computacionais capazes de sintetizar o conhecimento que os pesquisadores da área já dispões sobre o assunto. O modelo cognitivo deve simultaneamente atender os fundamentos descritos na teoria e ser simples bastante para ser computacionalmente factível. A simplificação do modelo não deve comprometer a sua usabilidade, ou seja, os resultados do modelo ainda devem ser dignos de confiança.

O desafio envolve o estudo de modelos já desenvolvidos pelos teóricos e sua adaptação a um objeto computacional. O modelo resultante deve ser capaz de ilustrar e mimetizar um funcionamento mental comprovável pela própria engenharia do sistema. Um dos principais desafios é superar a não observabilidade da cognição. A cognição é um processo interno ao cérebro com poucos indícios registráveis de sua atividade interna. Deve ser aplicado métodos científicos para acentuar e diferenciar estes estágios e deve ser feita uma cuidadosa investigação de todos os vestígios externos que possam retratar este funcionamento.

O propósito final é direcionar o modelo de entendimento da cognição para aplicação na melhoria do desempenho cognitivo das pessoas. A educação será a principal aplicação deste modelo, indicando os melhores caminhos de ensinar.

\section{O desafio e o problema de modelar a cognição}

A cognição é um campo vasto e complexo que pode abranger diversas visões e abordagens para se estudar e compreender. A proposta aqui procura delimitar o campo de estudo para que se possa desenvolver um modelo de engenharia com aplicações na área de educação. O problema aqui delimitado consiste no desenvolvimento de modelos computacionais de engenharia. Em um modelo de engenharia se encara a cognição como uma máquina e todo funcionamento cognitivo como uma execução de um processo nesta máquina. A modelagem é extremamente simplificada para minimamente suprir o entendimento e comportamento desejado.

$\mathrm{O}$ desenvolvimento mental pode ser considerado em vários escopos temporais. $\mathrm{O}$ modelo deve estabelecer o escopo de desenvolvimento mental almejado. Dentro do escopo temporal temos a granularidade do tempo como definição do passo considerado na avaliação do progresso cognitivo. O modelo mental pode então abarcar o desenvolvimento cognitivo ao longo de diversas gerações de indivíduos ou simplesmente considerar o avanço obtido em poucos segundos de esforço mental.

A aplicação do modelo mental tem um campo tão vasto quanto toda a extensão da atividade humana. Toda e qualquer atividade humana pode se beneficiar de um modelo que consiga rastrear o desenrolar das atividades cognitivas. Um modelo assim genérico exigiria uma vasta pesquisa e provavelmente se tornaria muito complexo de se lidar. O modelo cognitivo deve focar em uma área de aplicação para atingir uma configuração que é simultaneamente exequível e utilitária.

\subsection{Estudando a cognição}

Diversos teóricos já propuseram modelos cognitivos, existindo diversas fontes onde o estudo pode ser fundamentado. Os vários modelos teóricos existentes devem ser 
estudados, organizados e aplicados segundo às necessidades da pesquisa. Dentro do escopo temporal, as dimensões do espaço cognitivo devem ser equacionadas. Os modelos devem contemplar as dimensões do problema: a filogenética, a psicogenética e a microgenética.

O modelo dimensional é um requisito preliminar para transcrever os fundamentos teóricos na arquitetura da solução modelada. $O$ cérebro é uma convergência de diversas aquisições nos diversos domínios temporais. O cérebro do homo sapiens contém toda a história evolutiva da espécie. O indivíduo é a soma de tudo que aprendeu ao longo de sua vida, assim como de tudo que está pensando em um determinado instante. Todas estas dimensões delimitam o espaço de atuação dos processos cognitivos.

O pensamento do homem moderno, segundo Luria (1966a, 1966b) é dominado pela linguagem. Seminério também considera o processo cognitivo como uma cascata de linguagens. Em termos de computação, linguagens são processadas por sistemas de compiladores. Os modelos computacionais que mapeiam máquinas cognitivas podem então ser concebidos em forma de autômatos que interpretam uma cadeia de símbolos codificados representando o processamento cognitivo.

O espaço dimensional populado pelos casos de uso internos da máquina cognitiva constitui o cognoma humano. A expressão cognoma é análoga à expressão do genoma ou em melhor grau ao proteoma humano. A máquina cognitiva vai avançando por sequências de estados, formando expressões complexas associadas aos estágios do processamento mental. O cognoma é em suma a coleção de expressões cognitivas usadas na aquisição de todo e qualquer conhecimento ou habilidade.

\subsection{Acesso à cognição}

Toda a riqueza da máquina mental humana ainda está em aberto para ser investigada. Para abrir a caixa preta da mente é preciso contar com teorias que gerem hipóteses sobre o seu funcionamento interno. Os modelos cognitivos resultantes devem exercitar a mente para que produzam eventos marcadores dos estados internos. Os eventos que devemos observar nas dimensões cognitivas servirão para localizar o indivíduo no espaço dimensional (Marques, 2017).

Devido à dificuldade de acesso aos seus estados internos os estudos sobre a mente tendem a ter uma natureza behaviorista centrado em resposta a dados estímulos. No entanto, a falta de acesso aos estágios internos da cognição pode ser compensada por inferências sobre o comportamento observado. A diversidade de variações sobre as respostas esperadas é resultante do processamento interno necessário para produzi-las. Diversas abordagens disponíveis na ciência de dados devem ser aplicadas para discriminar estas evidências do funcionamento interno.

Em primeira instância deve ser modelada a máquina que representa o funcionamento básico do processo cognitivo. A máquina básica da cognição pode ser modelada por um autômato que codifica as aquisições sensoriais para uso interno da cognição. O tratamento de dados oriundos dos processos cognitivos é uma fonte de entendimento do funcionamento mental quando bem fundamentado por uma teoria que descreva com propriedade o engenho interno cognitivo. 
Uma vez que se tenha um modelo do autômato básico da cognição, a pesquisa pode prosseguir na investigação do uso que é feito deste autômato em diversas situações da aquisição do conhecimento. O rastreamento do funcionamento mental deve estudar os diversos estágios pelos quais o processamento cognitivo avança. Correlacionando os estágios com as situações de aprendizado propostas pode-se mapear o cognoma humano.

\section{Espaço de soluções do modelo cognitivo}

\subsection{Espaço dimensional temporal}

O espaço temporal considera os modelos conforme a granularidade do tempo de amostragem do desenvolvimento cognitivo. Este espaço delimita as estratégias de pesquisa principalmente voltadas para o indivíduo. Este espaço deve priorizar a diversidade de inteligências e ressaltar as diferenças individuais como patrimônio essencial da humanidade.

A dimensão filogenética estuda o desenvolvimento da cognição ao longo da formação da espécie. Embora esta dimensão é mais apropriada para o campo da Antropologia, dentro da Educação ela é importante para definir o status quo do cérebro atual. Neste sentido a modelagem cognitiva deve estudar as funções cerebrais inatas. Segundo Chomsky (2014) o inatismo determina a nossa capacidade de aprender e dominar a linguagem humana. Também podem ser consideradas nesta dimensão as funções executivas. Estas funções são nativas no cérebro, mas ainda podem variar em desempenho devido ao histórico de uso individual.

Ao longo da maturação cerebral do indivíduo temos a dimensão ontogenética ou psicogenética. As competências vão sendo habilitadas segundo o estágio de desenvolvimento biológico do cérebro. Nesta dimensão nós temos as etapas previstas por Piaget (1977). Em média, os primeiros estágios são habilitados com sucesso na população humana. A modelagem cognitiva deve-se concentrar no estágio formal e pós formal, que ainda são pouco difundidos e são fundamentais para se ter um indivíduo cognitivamente competente.

A microgênese é a dimensão onde se dá o processo interno de aprendizagem. $\mathrm{O}$ processo cognitivo se dá em um curto espaço de tempo e mesmo sem o indivíduo se dar conta do acontecido. Segundo Dehaene (2014), a maior parte deste processo cognitivo se dá inconscientemente por um conjunto maciço de processamento paralelo simultâneo. A modelagem desta máquina é a mais desafiadora, pois pouco se obtêm em dados que possam ser oferecidos pelo indivíduo pesquisado. Os dados sobre o funcionamento interno precisam ser extraídos por experimentos criteriosos e profundamente analisados.

\subsection{Espaço dimensional populacional}

O espaço populacional retrata a variação da cognição entre indivíduos de uma população. Este estudo deve delimitar as variações favoráveis e desfavoráveis. As favoráveis oferecem um diferencial ao indivíduo que o qualifica para uma posição social relevante. As desfavoráveis podem significar falhas no aparato cognitivo individual e devem ser analisadas e corrigidas. Os modelos cognitivos devem ser parametrizados 
para que possam preservar a diversidade da população, garantindo que todas as habilidades necessárias sejam cultivadas na educação.

A diversidade de inteligências é o principal legado que deve se preservar na espécie humana. A educação deve ser repensada para que as diferenças individuais sejam levadas em consideração. Os modelos cognitivos devem ser desenvolvidos com esta variabilidade em mente. Eles serão os facilitadores da personalização do processo educativo, evitando que o processo em massa deturpe o propósito de levar todos a um pleno desenvolvimento.

Dentro da diversidade do cognoma em uma população é possível que grupos característicos emerjam. A identificação destes grupos faz parte do estudo populacional. Estes grupos podem ser usados para melhorar o entendimento do cognoma, observando as diferenças entre eles. Tanto as diferenças como as semelhanças são pontos importantes na modelagem cognitiva. As diferenças marcam os limites da distribuição cognitiva em uma população. As semelhanças são marcadoras de especializações do cognoma que devem ser consideradas tanto na modelagem quanto no processo de ensino, que deve levar em conta estas peculiaridades.

\section{Avaliação e impacto de modelos cognitivos}

A avaliação dos modelos cognitivos se dá em três aspectos, o científico, o demográfico e o educacional. A avaliação científica do modelo se dará através da validação do seu isomorfismo com o comportamento humano descrito nos estudos atuais. A avaliação demográfica se dá no estudo da distribuição da cognição nas populações. Os resultados obtidos pelos modelos devem ser comparados com estatísticas disponíveis correspondentes. A avaliação mais relevante é aquela que implica o maior impacto social, no caso o aspecto educacional. Os modelos cognitivos são por construção auto avaliativos. Quando aplicados à educação devem avaliar a cognição dos indivíduos e propor novos caminhos para o ensino. O sucesso do indivíduo em seu desenvolvimento cognitivo será o marcador principal da aplicabilidade do modelo.

O impacto da modelagem cognitiva se dá em toda e qualquer atividade humana que exige a cognição. Um modelo cognitivo de sucesso pode estimar com grande acurácia o grau de aquisição de habilidades e conhecimentos.

\section{Obstáculos e dificuldades na aplicação de modelos cognitivos}

A principal dificuldade deste desafio é superar a baixa observabilidade dos processos cognitivos. Esta pesquisa irá requerer a formação de times interdisciplinares pouco disponíveis atualmente. Estes times deverão desenvolver soluções criativas para decifrar todo sinal disponível que possa dar detalhes sobre o funcionamento interno da mente.

Próteses computacionais ainda são olhados com alguma desconfiança. Modelos cognitivos são próteses de alto impacto e alta pervasividade e são, por estes motivos, passíveis de problemas de aceitação na sociedade humana. Neste aspecto, próteses cognitivas tem que ser validadas e testadas com grande cuidado e detalhe. Elas estarão em contato direto com seres humanos e devem, portanto, ser responsavelmente construídas e aplicadas. 


\section{Plano de estudo e aplicação do cognoma humano}

O plano de pesquisa com as Metas, Ações e Monitoramento é apresentado no Quadro 1.

Quadro 1: Metas, Ações e Monitoramento e Avaliação

\begin{tabular}{|c|c|c|c|}
\hline $\begin{array}{l}\text { Desafio ou } \\
\text { Problema }\end{array}$ & Metas & Ações & $\begin{array}{l}\text { Monitoramento e } \\
\text { Avaliação }\end{array}$ \\
\hline \multirow[t]{4}{*}{$\begin{array}{l}\text { Estudo e } \\
\text { validação de } \\
\text { modelos } \\
\text { cognitivos }\end{array}$} & $\begin{array}{c}\text { Produção de } \\
\text { modelos cognitivos }\end{array}$ & $\begin{array}{c}\text { Pesquisa e } \\
\text { desenvolvimento de } \\
\text { modelos da mente } \\
\text { humana. }\end{array}$ & $\begin{array}{l}\text { Produção científica } \\
\text { de material escrito } \\
\text { sobre os modelos }\end{array}$ \\
\hline & $\begin{array}{l}\text { Representações } \\
\text { Mentais }\end{array}$ & $\begin{array}{l}\text { Pesquisa usando os } \\
\text { modelos para capturar } \\
\text { representações }\end{array}$ & $\begin{array}{c}\text { Cruzamento de } \\
\text { representações } \\
\text { reconhecidas entre } \\
\text { times } \\
\end{array}$ \\
\hline & Idioma mental & $\begin{array}{c}\text { Aplicações de modelos } \\
\text { para decifrar trechos de } \\
\text { idiomas internos à } \\
\text { cognição }\end{array}$ & $\begin{array}{l}\text { Mapeamento de } \\
\text { trechos idiomáticos } \\
\text { contra testes } \\
\text { cognitivos }\end{array}$ \\
\hline & Captura conceitual & $\begin{array}{c}\text { Pesquisa da concatenação } \\
\text { de idiomas para observar } \\
\text { a formação de ideias } \\
\text { conceituais }\end{array}$ & $\begin{array}{l}\text { Mapeamento contra } \\
\text { testes pós formais }\end{array}$ \\
\hline \multirow[t]{3}{*}{$\begin{array}{l}\text { Consolidação dos } \\
\text { modelos } \\
\text { cognitivos no } \\
\text { âmbito individual. }\end{array}$} & $\begin{array}{l}\text { Dicionário de } \\
\text { verbetes } \\
\text { cognitivos, o } \\
\text { cognoma } \\
\text { representacional }\end{array}$ & $\begin{array}{l}\text { Desenvolvimento e } \\
\text { publicação de } \\
\text { instrumentos para } \\
\text { estimular o surgimento de } \\
\text { representações mentais }\end{array}$ & $\begin{array}{l}\text { Estudo da variação } \\
\text { estatística dos } \\
\text { dicionários obtidos } \\
\text { por times diversos }\end{array}$ \\
\hline & $\begin{array}{c}\text { Dicionário de idiomas } \\
\text { cognitivos, o } \\
\text { cognoma } \\
\text { idiomático }\end{array}$ & $\begin{array}{c}\text { Desenvolvimento e } \\
\text { publicação de } \\
\text { instrumentos para } \\
\text { estimular o encadeamento } \\
\text { de representações } \\
\text { mentais }\end{array}$ & $\begin{array}{l}\text { Estudo da variação } \\
\text { estatística dos } \\
\text { dicionários obtidos } \\
\text { por times diversos }\end{array}$ \\
\hline & $\begin{array}{c}\text { Atlas de conceitos e } \\
\text { redes } \\
\text { epistemológicas, } \\
\text { o cognoma } \\
\text { abstrato }\end{array}$ & $\begin{array}{l}\text { Estudo dos dicionários } \\
\text { produzidos pela } \\
\text { comunidade científica e } \\
\text { observação das } \\
\text { correlações }\end{array}$ & $\begin{array}{c}\text { Publicação de } \\
\text { cognomas referentes } \\
\text { a conceitos abstratos } \\
\text { observados }\end{array}$ \\
\hline \multirow[t]{4}{*}{$\begin{array}{l}\text { Consolidação dos } \\
\text { modelos } \\
\text { cognitivos no } \\
\text { âmbito } \\
\text { populacional }\end{array}$} & \multirow{2}{*}{$\begin{array}{c}\text { Contagem do uso do } \\
\text { cognoma nas } \\
\text { diversas } \\
\text { populações } \\
\text { cognitivas }\end{array}$} & $\begin{array}{l}\text { Especialização de times } \\
\text { em cada classe cognitivas } \\
\text { para levantamento dos } \\
\text { cognomas específicos }\end{array}$ & $\begin{array}{c}\text { Contribuição de } \\
\text { diversos times no } \\
\text { mapeamento do } \\
\text { cognoma } \\
\text { populacional }\end{array}$ \\
\hline & & $\begin{array}{l}\text { Conferências ou trilhas } \\
\text { específicas para o tema. }\end{array}$ & $\begin{array}{c}\text { Artigos sobre a } \\
\text { distribuição cognitiva }\end{array}$ \\
\hline & $\begin{array}{c}\text { Levantamento das } \\
\text { causas do desvio } \\
\text { cognitivo nas } \\
\text { populações } \\
\end{array}$ & $\begin{array}{l}\text { Caracterização cognitiva } \\
\text { das populações à luz dos } \\
\text { prognósticos dos teóricos }\end{array}$ & $\begin{array}{c}\text { Artigos sobre a } \\
\text { distribuição dos } \\
\text { ganhos e perdas } \\
\text { cognitivas }\end{array}$ \\
\hline & $\begin{array}{l}\text { Prescrição de ações } \\
\text { remediadoras }\end{array}$ & $\begin{array}{c}\text { Estudo do efeito de } \\
\text { práticas neuropedagógicas } \\
\text { na cognição }\end{array}$ & $\begin{array}{c}\text { Avaliações das } \\
\text { mudanças cognitivas } \\
\text { na população }\end{array}$ \\
\hline
\end{tabular}


Três grandes desafios são apresentados, seguido de suas metas, ações e do monitoramento e avaliação: (1) estudo e validação de modelos cognitivos; (2) consolidação dos modelos cognitivos no âmbito individual e (3) consolidação dos modelos cognitivos no âmbito populacional.

A validação do modelo requer que sejam modelados autômatos que simulem o processo cognitivo a contento. O modelo cognitivo validado pode ser usado no levantamento do cognoma humano. O cognoma deve ser a base para a modelação de próteses cognitivas a serem disseminadas nos diversos campos da atividade humana. $\mathrm{O}$ plano segue o modelo cognitivo de Seminério $(1984,1988)$, onde a linguagem L1 é o próprio autômato e os níveis representacional, idiomático e conceitual são respectivamente L2, L3 e L4. Cada uma destas linguagens-código vai exigir um esforço particular na concepção de experimentos que possam desvendar o seu significado.

O cognoma deve ser explorado em sua dimensão populacional. Os experimentos vão requerer a aplicação em vários indivíduos para que se possa capturar dados relevantes. O estudo das diferenças e semelhanças no cognoma em todos os níveis de linguagem código permitirá o fechamento dos modelos. Por fim os modelos deverão ser implementados como engenhos prostéticos que permitirão a captura, avaliação e intervenção na cognição humana. Modelos cognitivos e o conhecimento do cognoma serão uma importante ferramenta na educação e treinamento personalizado para se alcançar qualquer compreensão e competência desejada.

\section{Referências}

Chomsky, N. (2014), Aspects of the Theory of Syntax. Vol. 11. MIT press, 2014.

Dehaene, S. (2014), Consciousness and the Brain: Deciphering How the Brain Codes Our Thoughts, Penguin Book, 2014

Luria, A. R.; Tsvetkova, L. S. (1967) Les troubles de la resolution des problems. Paris: Gauthier-Villars.

Luria, A. R. (1966a) Higher Cortical Functions in Man. New York; Basic Books.

Luria, A. R. (1966b) Human Brain and Psychological Processes. New York, Harper.

Marques, C.V.M. (2017) EICA - Estruturas Internas Cognitivas Aprendentes: Um Modelo Neuro-Computacional Aplicado à Instância Psíquica do Sistemas Pessoa em Espaços Dimensionais. Tese de Doutorado. COPPE/SISTEMAS, Universidade Federal do Rio de Janeiro.

Piaget, J. (1977) Recherches sur l'abstraction réflechissante. Paris, Presses Universitaires de France.

Seminério, F. L. P. (1984) Infra-estrutura da cognição: fatores ou linguagens? Rio de Janeiro: FGV; ISOP.

(1988) Metaprocesso: a chave do desenvolvimento cognitivo: uma reavaliação da pedagogia contemporânea. Rio de Janeiro: FGV; ISOP. 